\title{
ESTIMATIVA DE PARÂMETROS BIOFÍSICOS DE PLANTIOS DE CAFÉ A PARTIR DE IMAGENS ORBITAIS DE ALTA RESOLUÇÃO ESPACIAL ${ }^{1}$
}

\author{
GLÁUCIA M. RAMIREZ ${ }^{2}$, JURANDIR ZULLO JÚNIOR ${ }^{3}$
}

\begin{abstract}
RESUMO: O uso de recursos de sensoriamento remoto orbital constitui um grande avanço no levantamento de dados sobre a cafeicultura, sobretudo por seu caráter temporal e baixo custo. Sendo assim, o objetivo do trabalho foi avaliar a utilização da imagem do satélite QUICKBIRD na determinação de parâmetros biofísicos importantes para a cultura cafeeira. Foram utilizados 25 talhões com plantios de café localizados entre os municípios de Ribeirão Corrente, Franca e Cristais Paulista (SP). Os parâmetros biofísicos utilizados foram os espaçamentos entre linhas e plantas, altura, IAF, diâmetro da copa, porcentagem de cobertura vegetal, rugosidade, variedade e biomassa. Foram utilizados valores de refletância real das bandas espectrais do satélite QUICKBIRD e os índices de vegetação NDVI, GVI, SAVI e RVI. A partir desses dados, foram feitas análises de regressão linear e não linear para a geração dos modelos de estimativa. A utilização de modelos de regressão baseados em equações não lineares mostrou-se mais adequado para determinar os parâmetros IAF e a porcentagem de biomassa, importantes como indicativos da produtividade da cultura cafeeira.
\end{abstract}

PALAVRAS-CHAVE: Quickbird, IAF, biomassa, altura, modelo.

\section{ESTIMATION OF BIOPHYSICAL PARAMETERS OF COFFEE FIELDS BASED ON HIGH-RESOLUTION SATELLITE IMAGES}

\begin{abstract}
Remote sensing data are each time more available and can be used to monitor the vegetal development of main agricultural crops, such as the Arabic coffee in Brazil, since that the relationship between spectral and agronomical data be well known. Therefore, this work had the main objective to assess the use of Quickbird satellite images to estimate biophysical parameters of coffee crop. Test area was composed by 25 coffee fields located between the cities of Ribeirão Corrente, Franca and Cristais Paulista (SP), Brazil, and the biophysical parameters used were row and between plants spacing, plant height, LAI, canopy diameter, percentage of vegetation cover, roughness and biomass. Spectral data were the reflectance of four bands of QUICKBIRD and values of four vegetations indexes (NDVI, GVI, SAVI and RVI) based on the same satellite. All these data were analyzed using linear and nonlinear regression methods to generate estimation models of biophysical parameters. The use of regression models based on nonlinear equations was more appropriate to estimate parameters such as the LAI and the percentage of biomass, important to indicate the productivity of coffee crop.
\end{abstract}

KEYWORDS: Quickbird, LAI, biomass, height, model.

\section{INTRODUÇÃO}

O café sempre foi sinônimo de progresso e contribuiu de maneira decisiva para a industrialização do País, sendo que, ainda hoje, é um dos seus produtos agrícolas mais importantes. Por ser um produto importante para a economia brasileira, a obtenção de informações precisas, em tempo hábil e com baixo custo, sobre a extensão e o rendimento da cultura, são instrumentos valiosos que podem viabilizar um planejamento adequado pela integração correta entre o mercado e a produção (LIU \& LIU, 1988). No Brasil, a obtenção de informações oficiais referentes à cultura

\footnotetext{
${ }^{1}$ Extraído da tese de doutorado do primeiro autor, financiada pelo CNPq.

${ }^{2}$ Universidade Federal de Lavras - UFLA, Caixa Postal 3037, Lavras - MG, glaucia_ramirez@ deg.ufla.br.

${ }^{3}$ CEPAGRI - Centro de Pesquisas Meteorológicas e Climáticas Aplicadas à Agricultura, UNICAMP, Campinas - SP, jurandir@cpa.unicamp.br. 
cafeeira (tais como área plantada, quantidade produzida e área colhida, por exemplo) é realizada pela CONAB (Companhia Nacional de Abastecimento) e IBGE (Instituto Brasileiro de Geografia e Estatística), que utilizam informações municipais obtidas através da aplicação de questionários padrões a produtores, cooperativas e representantes de órgãos públicos e privados. Segundo IPPOLITI-RAMILO et al. (1999) e COLLARES et al. (1993), os dados coletados, seguindo essa metodologia, são bastante suscetíveis a erros de análise humana, além de dificultar a estimativa da precisão dos dados e resultados obtidos. Outro problema relacionado à aquisição de dados da cafeicultura brasileira, segundo DUARTE \& BRITO (2007), é o alto custo financeiro e de pessoal demandados pela metodologia utilizada atualmente. Por isso, é necessária a ampliação de pesquisas que permitam identificar, quantificar e qualificar as áreas com plantios de café de maneira mais rápida, precisa e com custos menores.

Dentre as técnicas que podem ser consideradas nesse contexto, o sensoriamento remoto é uma das que possuem um grande potencial de aplicação, pois representa, segundo EPIPHANIO et al. (1994), um avanço significativo no levantamento de dados, no monitoramento e no planejamento agrícolas. Isso é devido, entre outros fatores, à ampliação do acesso aos dados de sensores remotos, à disponibilidade de equipamentos para o processamento dos dados que permitem estudar os diversos sistemas agrícolas e às dimensões continentais do País. Ressalta-se que a utilização de técnicas de sensoriamento remoto têm tido um aumento significativo no Brasil ao longo do tempo, nos mais diversos campos do conhecimento, de tal modo que o País pode ser considerado um dos maiores usuários mundiais de imagens e dados remotos. Segundo YI et al. (2007) e CAMPOS et al. (2004), o sensoriamento remoto permite obter informações objetivas para o acompanhamento e previsão de safras agrícola, uma vez que possibilita a identificação de áreas agrícolas e suas dinâmicas.

No caso específico da cafeicultura, trabalhos como os de BERTOLDO et al. (2003), ALVES \& LACERDA (2003), MOREIRA et al. (2003) e RAMIREZ et al. (2006) ilustram o potencial da aplicação do sensoriamento remoto no planejamento e desenvolvimento do setor. Apesar da importância destes e de outros trabalhos realizados, principalmente no Brasil, ainda são necessárias mais pesquisas que permitam fazer com que o sensoriamento remoto seja utilizado de maneira mais efetiva no levantamento, mapeamento e monitoramento de áreas cafeeiras, servindo de apoio aos métodos operacionais empregados atualmente. O conhecimento da correlação entre fatores biofísicos da cultura e sua resposta espectral podem contribuir de maneira efetiva no uso das imagens de alta resolução espacial para o cadastro da cultura e também podem auxiliar os métodos atuais de previsão e monitoramento de safras. O objetivo deste trabalho foi avaliar a utilização da imagem do satélite QUICKBIRD na determinação de parâmetros biofísicos, importantes na determinação da produtividade, e para o cadastro agrícola da cultura cafeeira.

\section{MATERIAL E MÉTODOS}

A área de estudo utilizada no trabalho está localizada na Região da Alta Mogiana, entre os municípios de Franca e Ribeirão Corrente, a noroeste do Estado de São Paulo, delimitada pelas coordenadas $20^{\circ} 26^{\prime}$ e $20^{\circ} 29^{\prime}$ de latitude sul e 47 $26^{\prime}$ e $47^{\circ} 34^{\prime}$ de longitude oeste. Esses municípios localizam-se em uma região tradicional de produção do café, possuindo altitude média de $860 \mathrm{~m}$ com relevo suavemente ondulado, clima tropical de altitude (Cwa, Köeppen) e predominância de Latossolo Vermelho-Escuro e Latossolo Roxo.

Dentro desses municípios, foi escolhida uma área de aproximadamente 600 ha em que foram selecionados 30 talhões com plantios de café para o levantamento dos parâmetros biofísicos e extração dos valores radiométricos registrados na imagem do QUICKBIRD (Figura 1). 


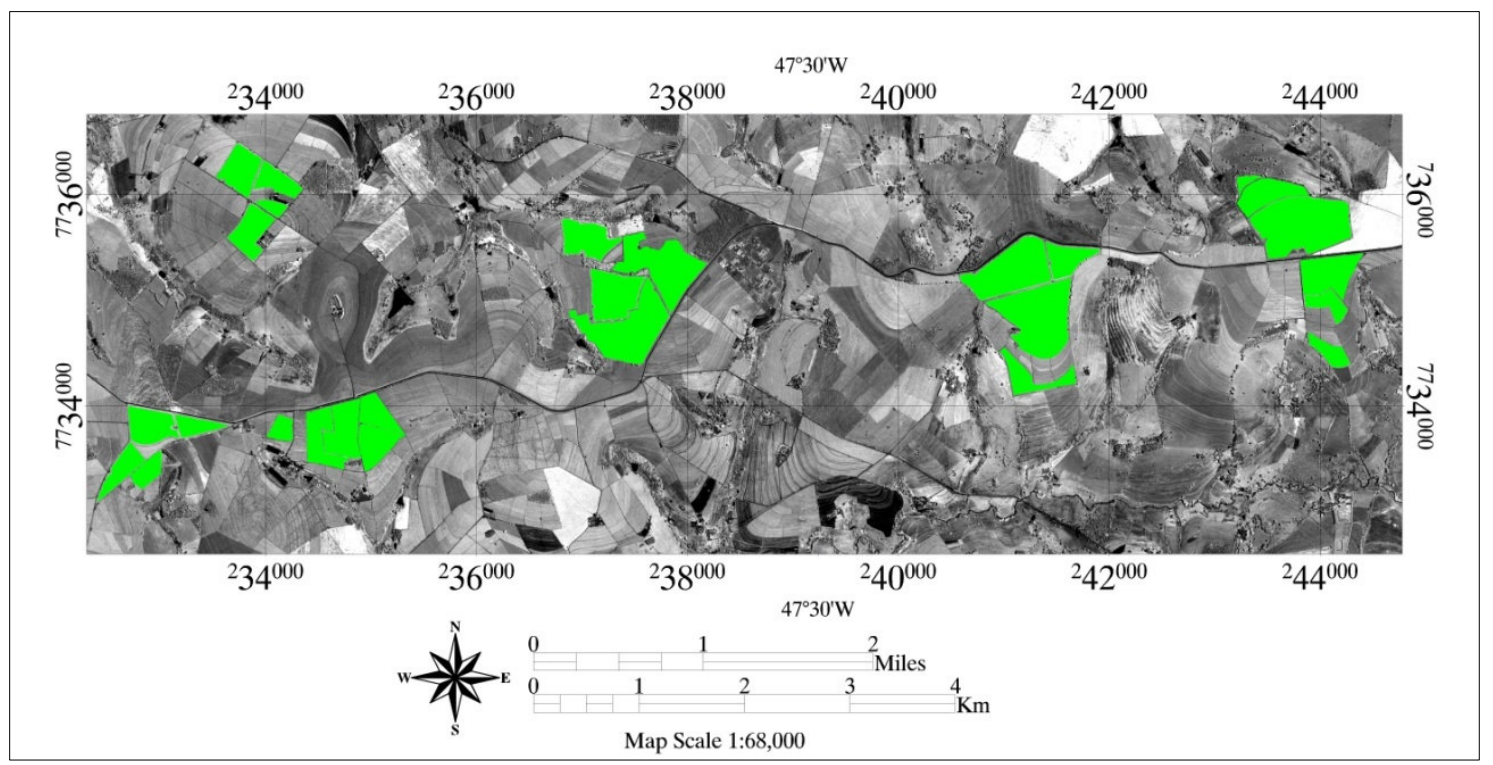

FIGURA 1. Imagem do satélite QUICKBIRD (Banda 4) mostrando os talhões selecionados para o trabalho. QuickBird satellite image (Band 4) showing the areas selected for this paper.

Os parâmetros levantados em campo e os valores calculados a partir destes são: altura de planta $(\mathrm{m})$, diâmetro de copa $(\mathrm{m})$, espaçamento entre linhas $(\mathrm{m})$, espaçamento entre plantas $(\mathrm{m})$, IAF $\left(\mathrm{m}^{2} \cdot \mathrm{m}^{-2}\right)$, porcentagem de cobertura do terreno pelas plantas de café $(\%)$, rugosidade $(\mathrm{m})$, densidade populacional (número de plantas $\mathrm{ha}^{-1}$ ) e biomassa $\left(\mathrm{tha}^{-1}\right)$. Os valores utilizados representam a média aritmética das medidas realizadas em 15 plantas por talhão.

O IAF foi calculado a partir da equação apresentada por FAVARIN et al. (2002), que utilizam a altura e o diâmetro da seção inferior do dossel (primeiro par de ramos), de acordo com a eq.(1):

$\mathrm{IAF}=0,0134+0,7276^{*} \mathrm{D}^{n} 2^{*} \mathrm{~h}$

em que,

D - diâmetro da seção inferior do dossel, m, e

$\mathrm{h}$ - altura do dossel, $\mathrm{m}$.

A rugosidade do dossel (Rugos) é representada pelo desvio-padrão dos valores das alturas medidas de acordo com PONZONI \& RESENDE (2004). Os valores das densidades populacionais (Dens. Pop.) foram calculados a partir dos espaçamentos (entre linhas e entre plantas) determinados em cada talhão, representando a quantidade de plantas existente em cada hectare. Para o cálculo da biomassa aérea, foi utilizada a fórmula desenvolvida por PÁSCUA (2002), de acordo com a eq.(2):

$$
\ln (\mathbf{B})=0,31543-0,87970 .(1 / \mathrm{h})
$$

em que,

$$
\begin{aligned}
& \text { B - biomassa seca, } \mathrm{t} \mathrm{ha}^{-1}, \mathrm{e} \\
& \mathrm{h} \text { - altura da planta, } \mathrm{m} .
\end{aligned}
$$

A percentagem de cobertura do terreno pelas plantas de café $(\% \mathrm{Cob})$ foi calculada a partir da área ocupada pelas plantas, de acordo com as eqs.(3) e (4):

- Para talhões com formação em renque (espaçamento entre plantas menor ou igual ao diâmetro médio das plantas) em que,

$$
\% \mathrm{COB}=(\mathrm{D} / \mathrm{EL}) 100
$$

D - diâmetro das plantas, m, e

EL - espaçamento entre linhas de plantio, $m$. 
- Para talhões onde o espaçamento entre plantas é maior que o diâmetro médio das plantas

$\% \mathrm{COB}=\left(\pi^{*} \mathrm{r}^{\wedge}\right)(\mathrm{EL} * \mathrm{EP})^{*} 100$

em que,

EL - espaçamento entre linhas de plantio, $\mathrm{m}$;

EP - espaçamento entre plantas na linha de plantio, $\mathrm{m}, \mathrm{e}$

$\mathrm{r}$ - raio médio das plantas medido em campo.

A imagem do QUICKBIRD foi adquirida no dia 5 de fevereiro de 2006, possuindo $69 \mathrm{~km}^{2}$, correspondendo à região de divisa entre os municípios de Ribeirão Corrente e Franca. A imagem possui quatro bandas espectrais, sendo três no visível (Q1-azul, $450 \mathrm{~nm}$ a $520 \mathrm{~nm}$; Q2-verde, $520 \mathrm{~nm}$ a $600 \mathrm{~nm}$; Q3-vermelho, $630 \mathrm{~nm}$ a $690 \mathrm{~nm}$ ) e uma no infravermelho próximo (Q4, $760 \mathrm{~nm}$ a $900 \mathrm{~nm}$ ), com resolução radiométrica de 16 bits e resolução espacial de 2,44 m.

Na imagem QUICKBIRD, foram feitas a correção atmosférica e a transformação dos níveis de cinza em valores de refletância real com a utilização do sistema computacional SCORADIS (Sistema de Correção Radiométrica de Imagens de Satélite), desenvolvido por ZULLO JÚNIOR (1994). Os parâmetros atmosféricos (espessura óptica dos aerossóis, coluna total de vapor d'água e carga total de ozônio), utilizados como entrada no programa SCORADIS, foram obtidos a partir do sensor MODIS seguindo a metodologia desenvolvida por NASCIMENTO (2006).

A partir das quatro bandas espectrais corrigidas (Q1, Q2, Q3 e Q4), foram calculados os índices de vegetação NDVI, SAVI, GVI e RVI. Todos os procedimentos necessários para o tratamento dos dados nas imagens foram realizados com a utilização do programa Envi 4.3.

Em seguida, obtiveram-se os valores médios da refletância e dos índices de vegetação para os 25 talhões amostrados. Esses valores foram exportados para o programa AJUSTE, desenvolvido por ZULLO JÚNIOR \& ARRUDA (1986).

O programa AJUSTE utiliza equações lineares e não lineares na análise de regressão e foi empregado no trabalho, visando a descrever o comportamento dos parâmetros biofísicos dos plantios de café com base nos dados espectrais obtidos através da imagem do QUICKBIRD. Segundo ZULLO JÚNIOR \& ARRUDA (1986), apesar de as análises de regressão linear ajustarem-se bem para vários tipos de dados, há muitos casos nas áreas biológica e agrícola que a relação não é linear e, portanto, a melhor representação para o fenômeno estudado não é uma reta. Por definição, um modelo de regressão é não linear se, pelo menos, um dos seus parâmetros aparecem de forma não linear.

Um modelo de regressão não linear é considerado "intrinsecamente linear" se este pode ser reduzido a um modelo linear por meio de uma parametrização apropriada. Pode-se, ainda, utilizar o termo "intrinsecamente linear" para referir-se a modelos que podem ser linearizados através de alguma transformação (MAZUCHELI \& ACHCAR, 2002). O programa AJUSTE utiliza 16 equações não lineares, que são linearizadas a partir das transformações apresentadas na Tabela 1.

Para avaliar os resultados obtidos nas análises de regressão, foram selecionados oito talhões com plantios de café localizados dentro da mesma área de estudo. Esses talhões foram escolhidos ao acaso e possuem distribuição espacial representativa para a área de trabalho, conforme apresentado na Figura 2. 
TABELA 1. Equações não lineares utilizadas na linearização das regressões empregadas no programa AJUSTE. Non-linear equations used in the linearization of the regressions applied in the AJUSTE program.

\begin{tabular}{l|c}
\hline Modelo & Equacões de Ajuste \\
\hline Senoidal & $y=a+b * \operatorname{sen}(0,017453 * x)$ \\
\hline Exponencial & $\ln y=\ln a+b x$ \\
\hline Recíproca & $1 / y=1 / a+b / a x$ \\
\hline Hiperbólica & $1 / y=a+b x$ \\
\hline Hiperbólica retangular & $\ln (1-y / c)=\ln a-b x$ \\
\hline Monomolecular & $\ln (c / y-1)=\ln a-b x$ \\
\hline Logística & $\ln (c / y-1)=-b * \ln a+b * \ln x$ \\
\hline Logística negativa & $\ln (\ln (c / y))=\ln a-b x$ \\
\hline Gompertz & $\log y=\log a+b * \log x+c(\log x)^{2}$ \\
\hline Alométrica linear & $y=a+b x+c x^{2}$ \\
\hline Alométrica curvilinear & $y=a+b x+c x^{2}+d x^{3}$ \\
\hline Quadrática & $\ln y=\ln a+b x+c x^{2}$ \\
\hline Cúbica & $\ln y=\ln a+b x+c x^{2}+d x^{3}$ \\
\hline Exponencial quadrática & $\ln y=\left(\ln a-\left(z^{2} / s^{2}\right)\right)+\left(2 z / s^{2}\right) x-\left(1 / s^{2}\right) x^{2}$ \\
\hline Exponencial cúbica &
\end{tabular}

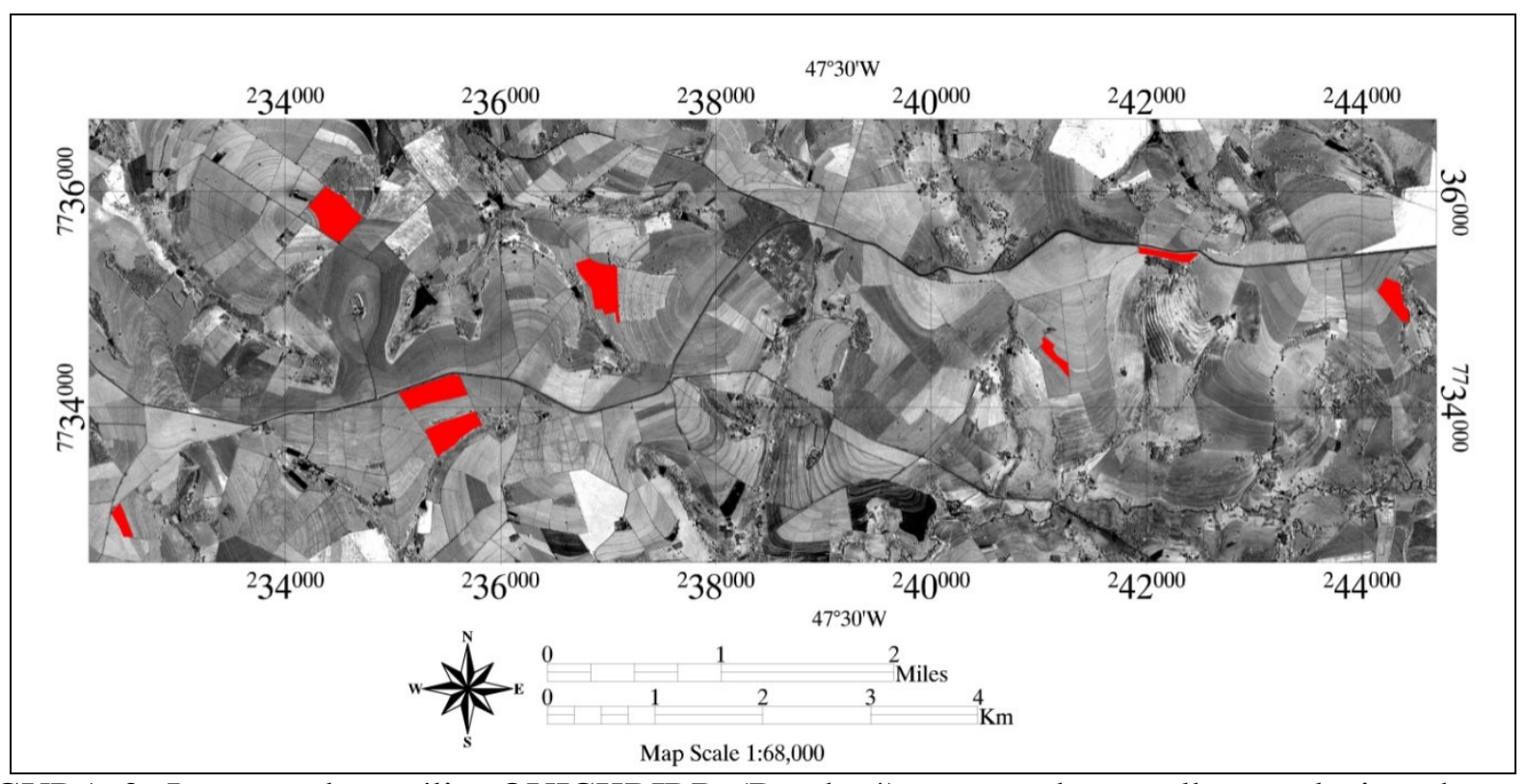

FIGURA 2. Imagem do satélite QUICKBIRD (Banda 4) mostrando os talhões selecionados para validação das equações obtidas através das análises de regressão. QuickBird satellite image (Band 4) showing the areas selected for validation of the equations obtained through regression analysis.

A sequência metodológica utilizada para a obtenção dos parâmetros biofísicos, a partir da imagem do QUICKBIRD, é apresentada completamente na Figura 3. 


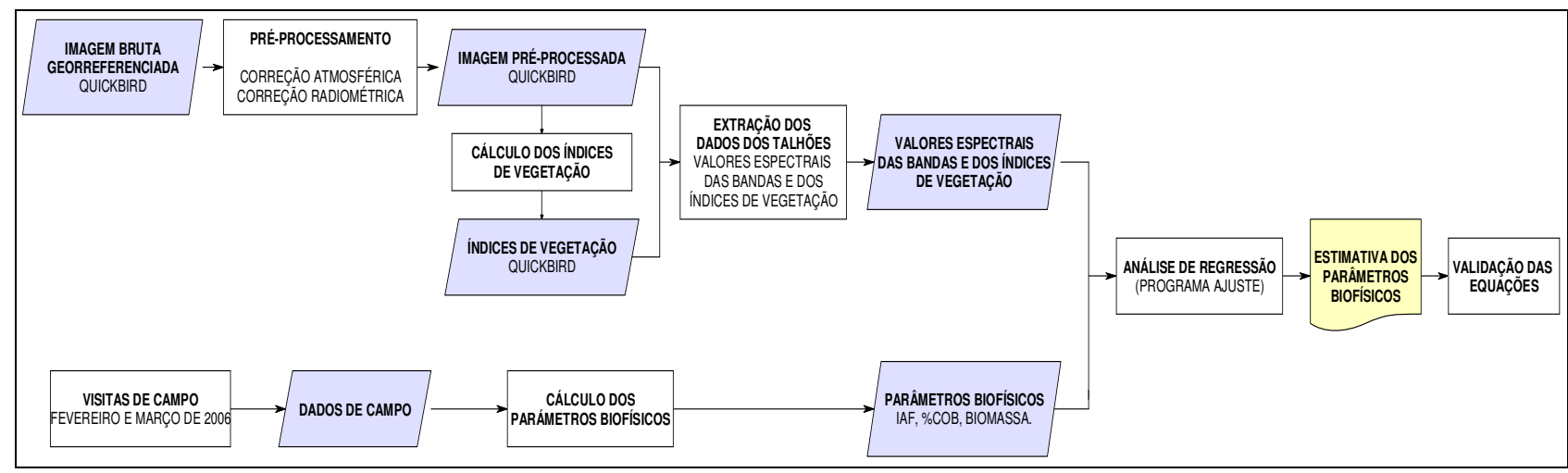

FIGURA 3. Metodologia utilizada para o desenvolvimento do trabalho. Methodology applied to develop this paper.

\section{RESULTADOS E DISCUSSÃO}

Na Tabela 2, apresentam-se os dados levantados em campo e os respectivos parâmetros biofísicos determinados a partir deles.

TABELA 2. Descrição dos valores médios dos parâmetros biofísicos nos 25 talhões amostrados em campo. Description of average values of the biophysical parameters in the 25 areas sampled in the field.

\begin{tabular}{c|c|c|c|c|c|c|c|c|c|c}
\hline Talhão & Varied & $\begin{array}{c}\text { EL } \\
(\mathrm{m})\end{array}$ & $\begin{array}{c}\text { EP } \\
(\mathrm{m})\end{array}$ & $\begin{array}{c}\text { Diâm } \\
(\mathrm{m})\end{array}$ & $\begin{array}{c}\text { Alt } \\
(\mathrm{m})\end{array}$ & $\begin{array}{c}\text { IAF } \\
\left(\mathrm{m}^{2} \mathrm{~m}^{-2}\right)\end{array}$ & $\begin{array}{c}\text { \%Cob } \\
(\mathrm{m})\end{array}$ & $\begin{array}{c}\text { Bio } \\
\left(\mathrm{tha}^{-1}\right)\end{array}$ & $\begin{array}{c}\text { Dens } \\
\left(\mathrm{pl} \mathrm{ha}^{-1}\right)\end{array}$ & $\begin{array}{c}\text { Rugos } \\
(\mathrm{m})\end{array}$ \\
\hline 2 & MN & 4,0 & 1,0 & 1,3 & 1,9 & 2,1 & 33,3 & 0,90 & $2.500,0$ & 0,3 \\
3 & MN & 3,0 & 1,0 & 1,8 & 1,8 & 3,7 & 60,0 & 0,89 & $3.333,3$ & 0,1 \\
4 & MN & 3,0 & 1,3 & 2,1 & 2,7 & 6,8 & 69,0 & 0,99 & $2.564,1$ & 0,5 \\
6 & MN & 1,5 & 0,7 & 1,2 & 2,1 & 4,6 & 80,7 & 0,92 & $9.523,8$ & 0,2 \\
A & MN & 3,0 & 0,9 & 2,1 & 2,4 & 6,3 & 71,3 & 0,95 & $3.921,6$ & 0,3 \\
Ac & Catuaí & 2,0 & 0,7 & 1,9 & 1,7 & 4,1 & 96,0 & 0,86 & $7.692,3$ & 0,1 \\
B & Catuaí & 3,8 & 0,6 & 2,1 & 2,1 & 5,4 & 55,0 & 0,92 & $4.386,0$ & 0,1 \\
D & Catuaí & 4,0 & 0,9 & 1,5 & 2,0 & 3,4 & 38,5 & 0,92 & $2.941,2$ & 0,1 \\
E1 & MN & 3,8 & 1,0 & 2,6 & 2,2 & 7,5 & 68,1 & 0,93 & $2.631,6$ & 0,2 \\
F1 & MN & 3,8 & 1,0 & 2,2 & 2,2 & 6,1 & 57,8 & 0,94 & $2.631,6$ & 0,2 \\
G1 & MN+Cat. & 2,0 & 1,0 & 1,9 & 2,1 & 4,8 & 95,0 & 0,92 & $5.000,0$ & 0,4 \\
H & Catuaí & 3,5 & 0,8 & 2,2 & 2,2 & 6,0 & 62,9 & 0,93 & $3.571,4$ & 0,1 \\
I & MN & 3,5 & 0,8 & 2,1 & 2,1 & 5,3 & 58,6 & 0,92 & $3.571,4$ & 0,3 \\
J & Catuaí & 3,0 & 0,7 & 2,2 & 2,3 & 6,3 & 72,0 & 0,95 & $4.761,9$ & 0,3 \\
L & Catuaí & 3,0 & 1,0 & 1,9 & 2,0 & 4,7 & 64,0 & 0,91 & $3.333,3$ & 0,2 \\
O & Catuaí & 3,2 & 0,7 & 1,9 & 2,1 & 4,8 & 58,1 & 0,93 & $4.464,3$ & 0,2 \\
P & Catuaí & 3,0 & 1,0 & 2,0 & 2,3 & 5,5 & 66,7 & 0,94 & $3.333,3$ & 0,2 \\
Q & MN & 3,5 & 0,8 & 1,7 & 2,2 & 4,2 & 47,5 & 0,93 & $3.809,5$ & 0,1 \\
R & Catuaí & 4,0 & 2,0 & 2,1 & 2,1 & 5,7 & 52,9 & 0,93 & $1.250,0$ & 0,3 \\
S & MN & 4,0 & 1,3 & 2,0 & 2,8 & 6,9 & 50,4 & 1,00 & $2.000,0$ & 0,2 \\
T & MN & 4,0 & 1,3 & 2,3 & 2,6 & 7,5 & 58,4 & 0,97 & $2.000,0$ & 0,3 \\
U & Catuaí & 4,0 & 1,5 & 2,1 & 2,4 & 6,2 & 52,7 & 0,96 & $1.666,7$ & 0,4 \\
V & Catuaí & 3,5 & 0,8 & 0,7 & 0,9 & 0,1 & 14,0 & 0,61 & $3.809,5$ & 0,1 \\
X & MN & 3,5 & 0,8 & 0,9 & 1,3 & 0,7 & 24,5 & 0,76 & $3.809,5$ & 0,1 \\
Y & MN & 3,5 & 0,9 & 1,8 & 2,6 & 5,5 & 51,6 & 0,98 & $3.361,3$ & 0,2 \\
\hline MN
\end{tabular}

MN (variedade Mundo Novo); EL (espaçamento entre linhas de plantio); EP (espaçamento entre plantas na linha de plantio); Diâm (diâmetro das plantas); Alt (altura); IAF (índice de área foliar); \%Cob (percentagem de cobertura do terreno pelas plantas); Bio (biomassa); Dens (densidade populacional), e Rugos (rugosidade). 
A partir dos dados apresentados na Tabela 2 e dos valores médios das bandas espectrais e dos índices de vegetação, foram obtidas as equações e seus respectivos $\mathrm{R}^{2}$ para cada parâmetro biofísico, banda espectral e índice de vegetação utilizado como entrada no programa AJUSTE. Foram consideradas regressões com valores de $\mathrm{R}$ maiores que 0,85 entre os parâmetros biofísicos (variável dependente) e as bandas e índices de vegetação gerados a partir dos dados do QUICKBIRD (variável independente). Para avaliar os resultados das análises de regressão, foram gerados gráficos de dispersão dos pontos mostrando a linha de ajuste. A equação obtida foi aplicada nos oito talhões-teste separados para este fim.

$\mathrm{Na}$ Tabela 3, são apresentadas as equações de ajuste e seus respectivos $\mathrm{R}^{2}$ para os parâmetros calculados a partir da imagem do QUICKBIRD.

TABELA 3. Equações geradas pelo programa Ajuste e seus respectivos $\mathrm{R}^{2}$ para os parâmetros Biomassa, IAF e Altura. Equations generated by the AJUSTE program and their respective $R^{2}$ for the parameters biomass, LAI and height.

\begin{tabular}{|c|c|}
\hline $\begin{array}{l}\text { Equação } \\
\end{array}$ & $\mathrm{R}^{2}(\%)$ \\
\hline $\mathrm{Bio}=4,47563434 \cdot 10^{-006} *\left(\mathrm{Q}^{-8,62882805+(-3,49179101) * \text { LOG10(Q2)})}\right.$ & 85,5 \\
\hline $\mathrm{Bio}=0,557779312^{*} \operatorname{EXP}\left(83,6939774^{*} \mathrm{Q} 1+(-3871,16577) *\left(\mathrm{Q1}^{2}\right)+(45794,7969) *\left(\mathrm{Q1}^{3}\right)\right)$ & 84,2 \\
\hline $\mathrm{IAF}=1,24841582^{*} 10^{-38} *\left(\mathrm{Q}^{-62,3811951+(-25,1502056) \operatorname{LOG}(\mathrm{X})}\right)$ & 76,0 \\
\hline $\mathrm{IAF}=0,186532617 * \operatorname{EXP}\left(542,184448 * \mathrm{Q} 1+(-25258,4805) *\left(\mathrm{Q}^{2}\right)+(292005,594) *\left(\mathrm{Q}^{3}\right)\right)$ & 76,0 \\
\hline $\mathrm{Alt}=0,000270756^{*} \mathrm{EXP}\left(-382,140381^{*} \mathrm{Q} 2+(-5112,61768)^{*}\left(\mathrm{Q}^{2}\right)+(20986,9004)^{*}\left(\mathrm{Q}^{3}\right)\right)$ & 72,4 \\
\hline
\end{tabular}

Bio - biomassa; IAF - índice de área foliar; Alt - altura; Q1 - banda 1 (azul) do satélite QUICKBIRD); Q2 - banda 2 (verde) do satélite QUICKBIRD.

O parâmetro Biomassa foi o mais bem correlacionado com os dados do satélite, de acordo com as equações não lineares utilizadas no programa AJUSTE. A Biomassa foi ajustada às bandas Q1 e Q2 do QUICKIBIRD. O ajuste do parâmetro biomassa com a banda Q2 foi o que obteve o maior $\mathrm{R}^{2}(85 \%)$, sendo que a equação que proporcionou o melhor ajuste foi a alométrica curvilinear.

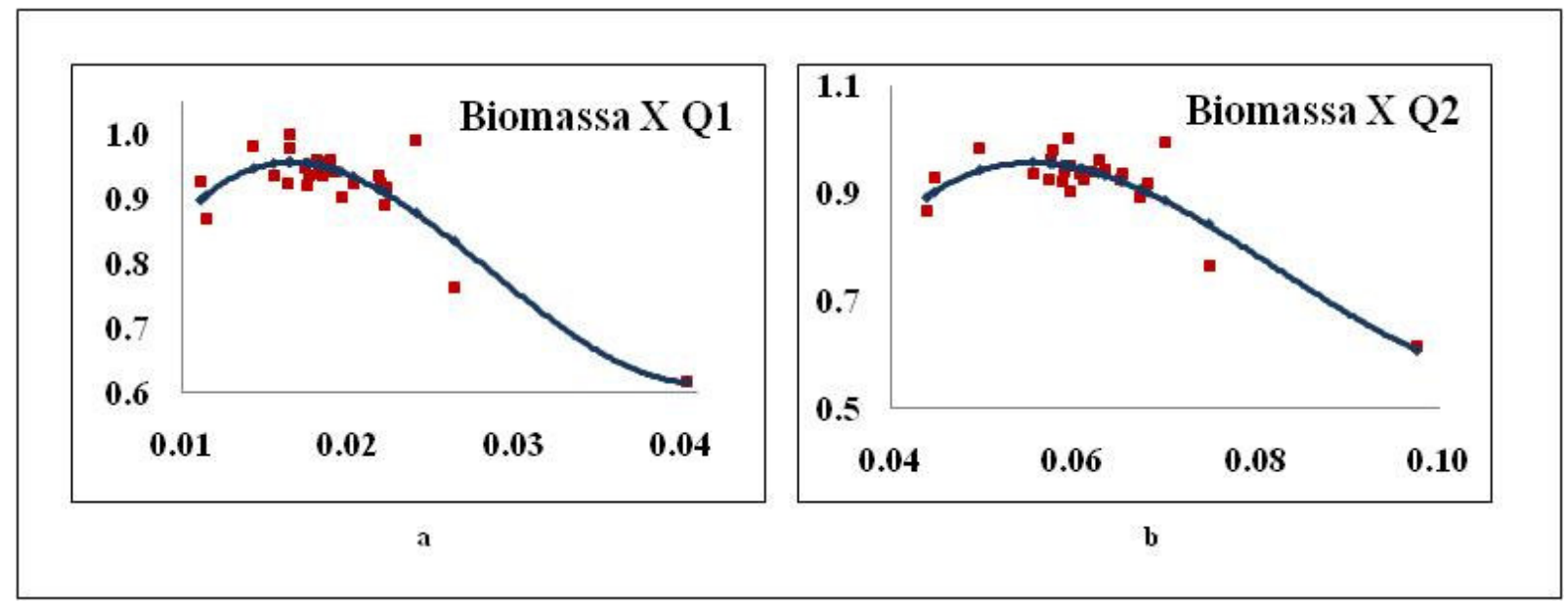

FIGURA 4. Dispersão dos dados de biomassa e as curvas de ajuste desses pontos para as bandas $1 \mathrm{e}$ 2 do satélite QUICKBIRD. Dispersion of the biomass data and the adjustment curve of these points to the bands 1 and 2 of the QuickBird satellite.

Esse resultado vem corroborar os obtidos por BAUSCH et al. (2008), que encontraram o maior coeficiente de correlação com a Biomassa $\left(\mathrm{R}^{2}=0,59\right)$, quando foi utilizada a banda 2 do QUICKBIRD em um estudo com milho irrigado. 
Na Figura 5, são mostrados os resultados da análise das equações realizada com os talhões-teste.

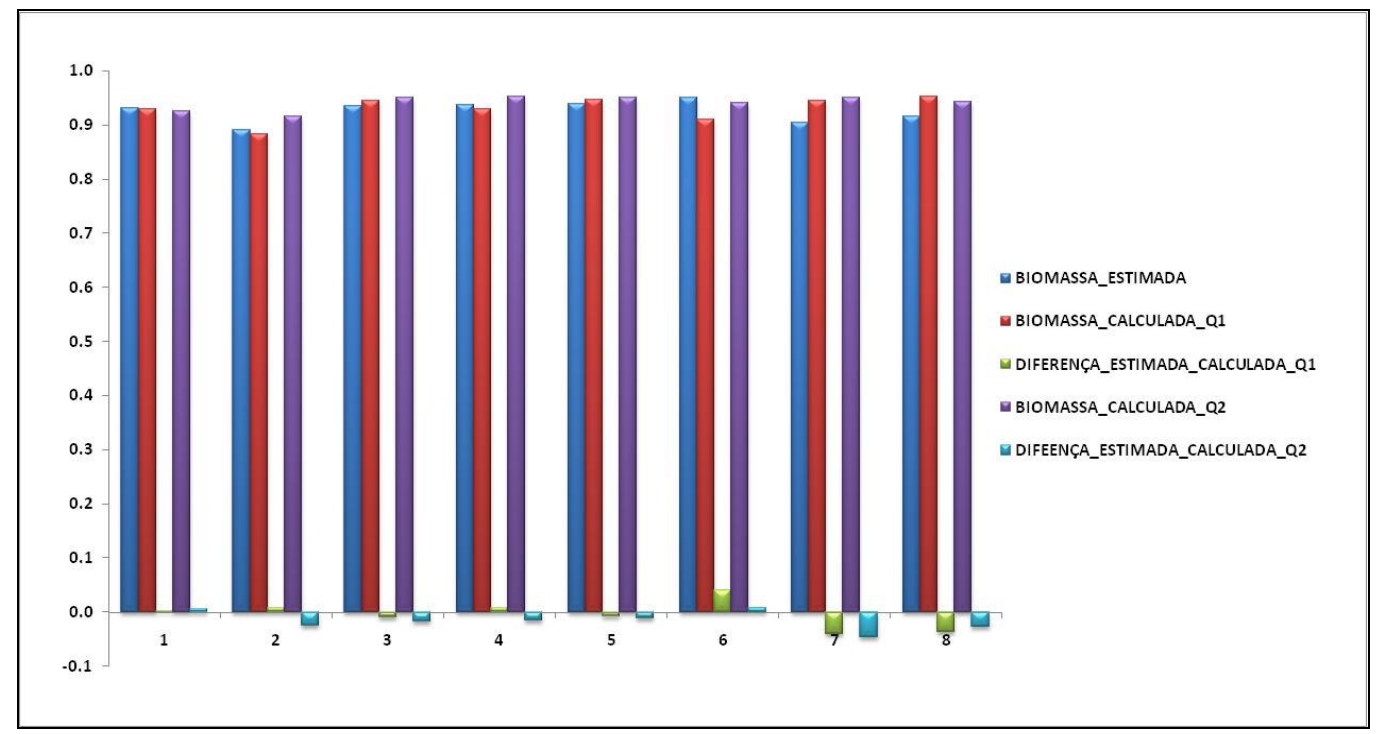

FIGURA 5. Valores de Biomassa estimados, calculados e da diferença existente entre eles, para as bandas 1 e 2 (Q1 e Q2, respectivamente) do satélite QUICKBIRD. Biomass Values estimated, calculated, and difference between them, for bands 1 and 2 (Q1 and Q2, respectively) of the QuickBird satellite.

O maior erro relativo foi de 4,4\% para Q1 e 5,1\% para Q2, enquanto o menor erro foi de $0,3 \%$ para Q1 e de 0,6\% para Q2. O erro relativo médio foi de 2,1\% para as duas bandas. Desse modo, os modelos de regressão gerados para o parâmetro Biomassa apresentaram bons resultados, com coeficientes de correlação elevados e valores baixos para os erros relativos médios, mostrando-se promissor para a estimativa da Biomassa em plantios de café. O parâmetro IAF mostrou-se bem correlacionado tanto com a banda 1 como com a banda 2, sendo que a equação que apresentou melhor resultado para a banda 1 foi a exponencial cúbica, e para a banda 2, foi a equação alométrica curvilinear. Observam-se, na Figura 6, as curvas de dispersão dos dados e a curva de melhor ajuste para eles, sendo que os valores do coeficiente de correlação $\left(\mathrm{R}^{2}\right)$ foram iguais $(76 \%)$ nos dois casos.

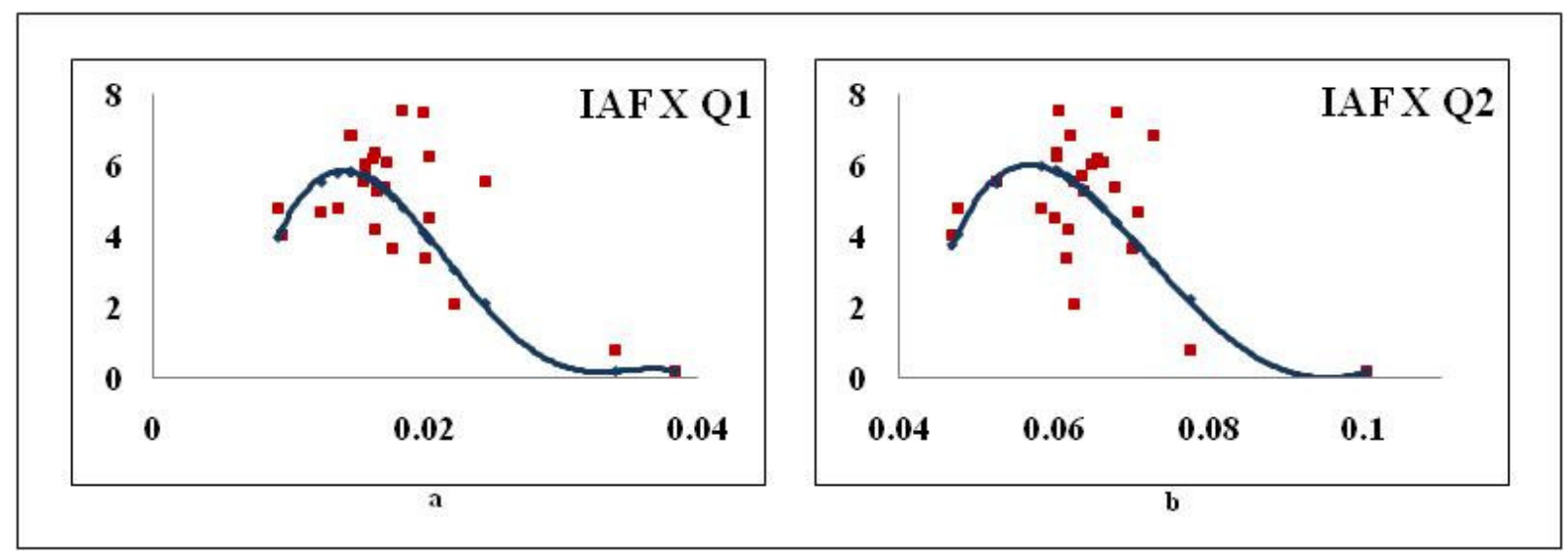

FIGURA 6. Dispersão dos dados de IAF e as curvas de ajuste desses pontos para as bandas 1 e 2 do satélite QUICKBIRD. Dispersion of the LAI data and the adjustment curve of these points to the bands 1 and 2 satellite QuickBird.

WU et al. (2007), avaliando a eficiência de índices de vegetação (IV) no cálculo do índice de área foliar (IAF), em imagens QUICKBIRD, obtiveram valores de $\mathrm{R}^{2}$ iguais a 0,63 para a cultura do 
milho e de 0,79 para a batata. Apesar de o coeficiente da batata ser maior que o obtido neste trabalho, ressalta-se que essa cultura possui característica agronômica muito diferente da cultura cafeeira. Por não haver referências bibliográficas sobre a utilização de imagens de alta resolução espacial no estudo de parâmetros biofísicos de cafezais, foi preciso comparar os resultados obtidos com outros tipos de cultura.

COLOMBO et al. (2003) obtiveram resultados significativos utilizando valores de NDVI da imagem IKONOS para determinar o IAF em vários tipos de cobertura vegetal. Para área de floresta, que possui mais semelhança com áreas cafeeiras, o coeficiente de determinação só foi significativo $(0,73)$ quando se considerou a textura da imagem pancromática.

Ressalta-se que os índices de vegetação avaliados neste trabalho não obtiveram resultados significativos com nenhum dos parâmetros biofísicos avaliados, somente as bandas 1 e 2 e os parâmetros IAF, biomassa e altura obtiveram resultados significativos.

Na Figura 7, são mostrados os resultados da avaliação das equações realizadas com os talhões-teste entre as bandas Q1 e Q2 e o IAF. O maior erro relativo obtido foi de 34,1\% para Q1 e $54,4 \%$ para Q2, sendo que, em ambos os casos, o talhão com maior erro possuía o menor IAF estimado. O menor erro relativo encontrado foi 2,58\% para Q1 e 2,1\% para Q2, sendo que o menor erro relativo médio foi obtido com a banda 1 (17\%). Q2 apresentou erro relativo médio de 21,3\%.

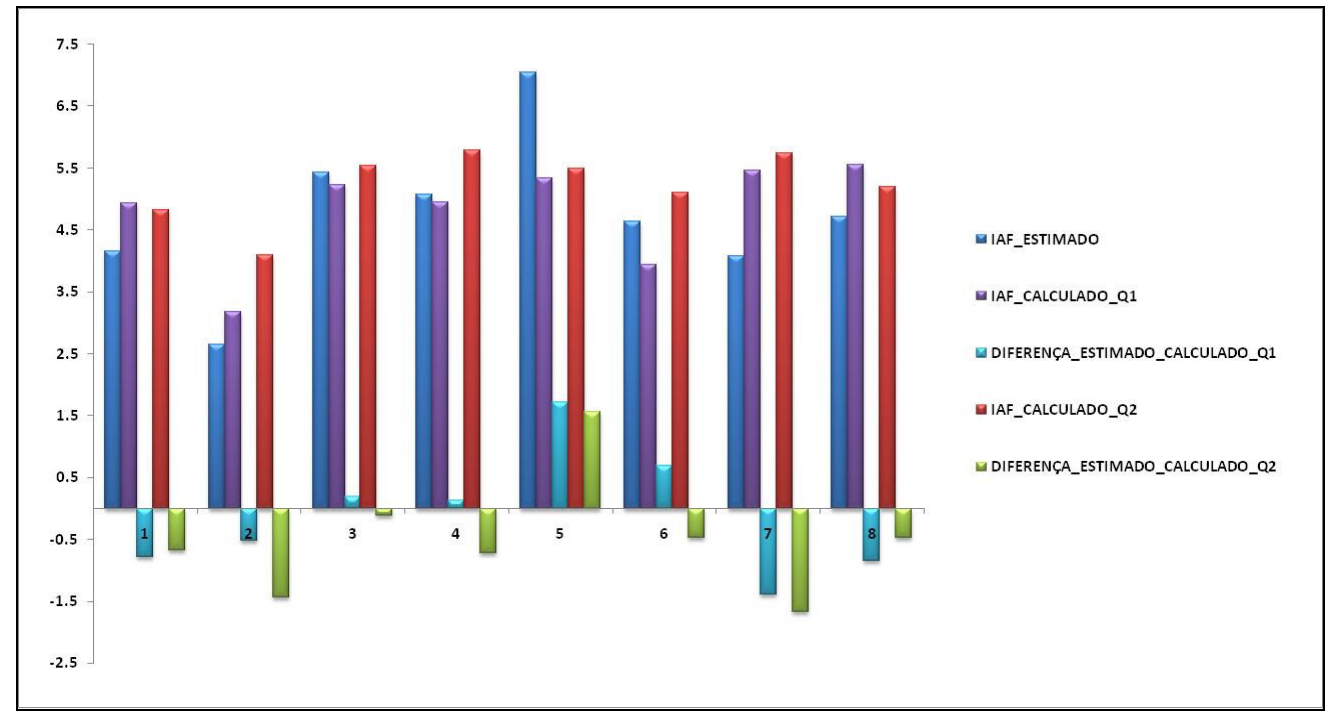

FIGURA 7. Valores de IAF estimados, calculados e da diferença existente entre eles, para as banda 1 e 2 (Q1 e Q2, respectivamente) do satélite QUICKBIRD. LAI Values estimated, calculated, and difference between them, for bands 1 and 2 (Q1 and Q2, respectively) of the QuickBird satellite.

O parâmetro altura foi correlacionado somente com a banda 2 do QUICKBIRD $(72,4 \%)$, sendo que a equação exponencial cúbica foi a que propiciou o melhor ajuste. Observa-se, na Figura 8, a curva de dispersão dos dados e a curva de melhor ajuste para eles.

Na Figura 9, são mostrados os resultados da avaliação das equações realizada com os talhõesteste para o parâmetro altura.

O maior erro relativo foi de $18,6 \%$, e o menor, de 1,1\%. O erro relativo médio foi de 7,6\%, sendo que os valores das alturas foram, de modo geral, superestimados pelo modelo de regressão.

Embora o programa AJUSTE utilize também a equação linear, em nenhum dos casos avaliados ela teve correlação alta com os parâmetros avaliados, sendo que, na maioria dos casos, o $\mathrm{R}^{2}$ ficou abaixo de 0,30 . Isto vem corroborar a afirmação feita por ZULLO JÚNIOR \& ARRUDA (1989) que a relação existente entre dados experimentais na área agrícola normalmente não é linear. 


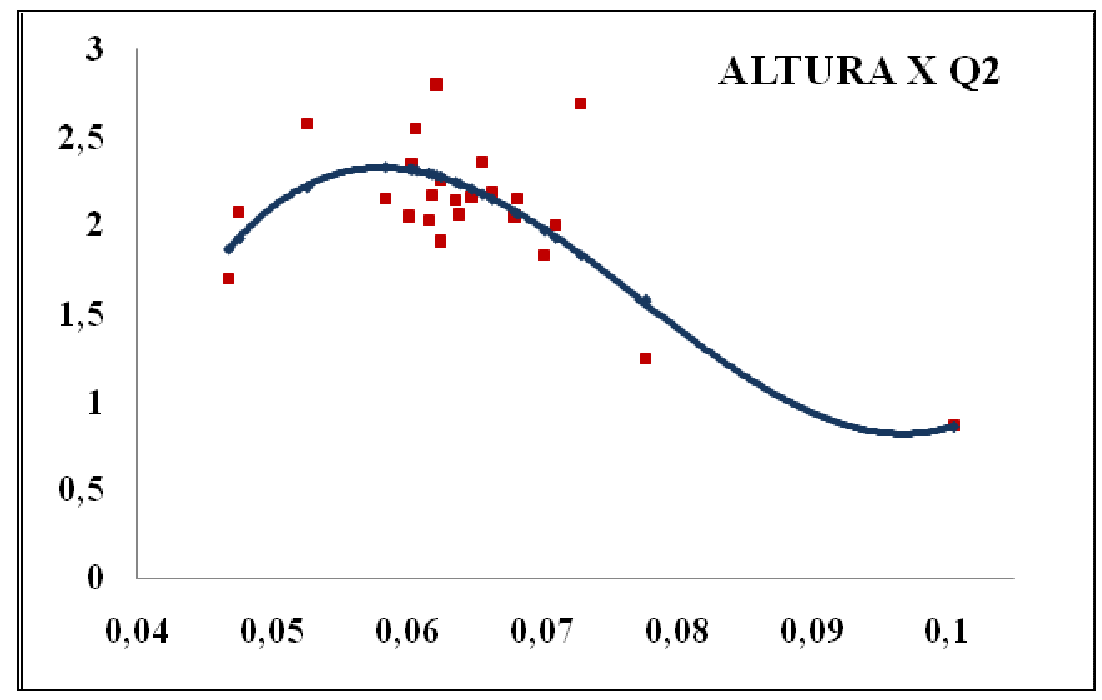

FIGURA 8. Dispersão dos dados de altura e as curvas de ajuste desses pontos para as bandas 1 e 2 do satélite QUICKBIRD. Dispersion of the height data and the adjustment curve of these points to the bands 1 and 2 satellite QuickBird.

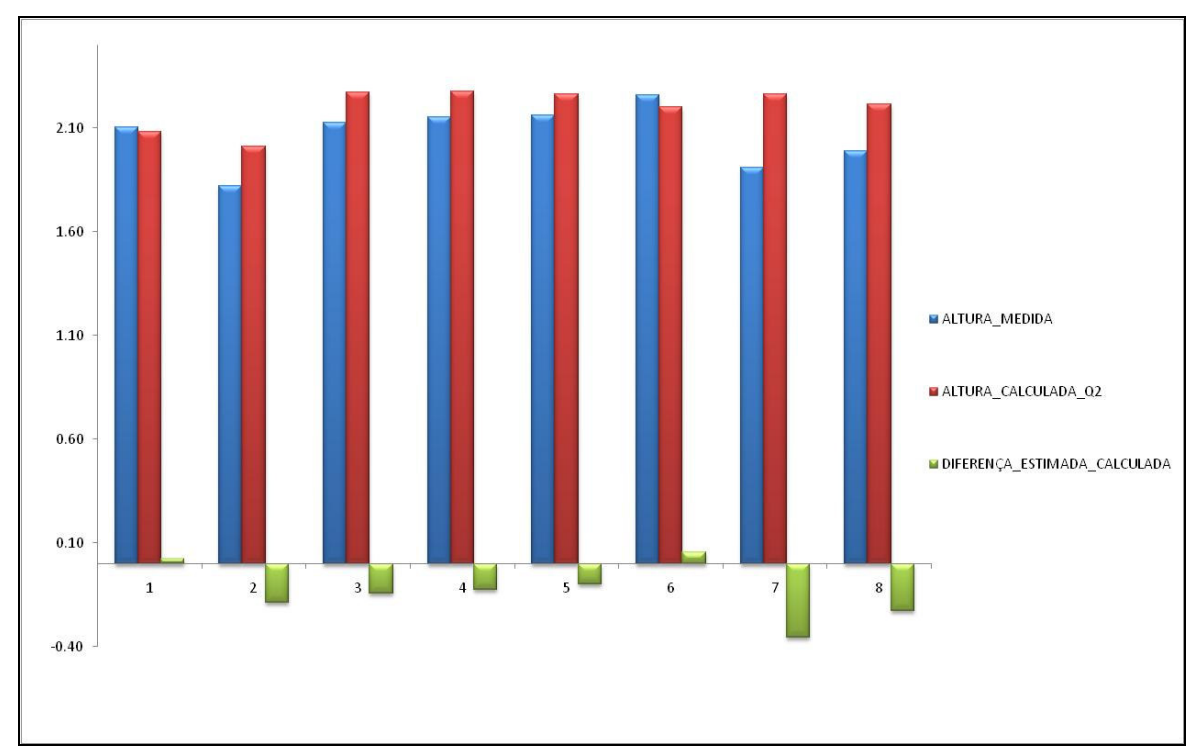

FIGURA 9. Valores de altura medidos, calculados e da diferença existente entre eles, para a banda 2 (Q2) do satélite QUICKBIRD. Height values estimated, calculated, and difference between them, for bands 1 and 2 (Q1 and Q2, respectively) of the QuickBird satellite.

\section{CONCLUSÕES}

Os parâmetros biofísicos altura, índice de área foliar e a biomassa de plantios de café podem ser estimados, utilizando-se das bandas espectrais do azul e do verde do satélite QUICKBIRD, através da utilização de modelos de regressão baseados em equações não lineares.

Os índices de vegetação NDVI, SAVI, GVI e RVI, calculados a partir da imagem QUICKBIRD, não se mostraram bons estimadores dos parâmetros biofísicos de cafezais.

O uso de imagens de alta resolução espacial mostra-se bastante promissor no estudo de áreas cafeeiras, uma vez que permite detectar características biofísicas importantes para acompanhamento das safras da cultura. 


\section{REFERÊNCIAS}

ALVES, H.M.R.; LACERDA, M.P.C. Caracterização de agroecossistemas cafeeiros nas principais regiões produtoras no Estado de Minas Gerais. In: SIMPÓSIO BRASILEIRO DE

SENSORIAMENTO REMOTO, 2003, Belo Horizonte. Anais... Belo Horizonte: INPE, 2003. p.1117.

BAUSCH, W.C.; HALVORSON, A.D.; CIPRA, J. Quickbird satellite and ground-based multispectral data correlations with agronomic parameters of irrigated maize grown in small plots. Biosystems Engineering, v.101, n.1, p. 306-315, 2008. Disponível em $<$ http://www.sciencedirect.com>. Acesso em: 25 out. 2008.

BERTOLDO, M.A.; ALVES, H.M.R.; VIEIRA, T.G.C.; OLIVEIRA, M.R.; MARQUES, H. Caracterização da cultura cafeeira em relação às classes de solos e declividade, utilizando técnicas de geoprocessamento na região de São Sebastião do Paraíso - MG. In: SIMPÓSIO BRASILEIRO DE SENSORIAMENTO REMOTO, 11., 2003, Belo Horizonte. Anais... São José dos Campos: INPE, 2003. 1 CD-ROM

CAMPOS, S.; SILVA, M.; PIROLI, E.L.; CARDOSO, L.G.; BARROS, Z.X. Evolução do uso da terra entre 1996 e 1999 no município de Botucatu - SP. Engenharia Agrícola, Jaboticabal, v.24, n.1, p.211-218, 2004. Disponível em: <http://www.scielo.br/pdf/eagri/v24n1/v24n01a24.pdf>. Acesso em: 29 abr. 2009.

COLLARES, J.E.R.; LAURIA, C.A.; CARRILHO, M.M. Pesquisa de previsão e acompanhamento de safras baseada em painéis de amostras de áreas. In: SIMPÓSIO BRASILEIRO DE

SENSORIAMENTO REMOTO, 7., 1993, Curitiba. Anais... Curitiba: INPE, 1993. p.450-453.

COLOMBO, R.; BELLINGERI, D.; FASOLINI, D.; MARINO, C.M. Retrieval of leaf area index in different vegetation types using high resolution satellite data. Remote Sensing of Environment, New York, v.86, p.120-131, 2003.

DUARTE, W.O.; BRITO, J.L.N.S. Mapeamento da Cultura do Café no município de ARAGUARIMG utilizando imagens do Sensor CCD do satélite CBERS-2. In: SIMPÓSIO BRASILEIRO DE SENSORIAMENTO REMOTO, 13., 2007, Florianópolis. Anais... São José dos Campos: INPE, 2007. p.161-167.

EPIPHANIO, J.C.N.; LEONARDI, L.; FORMAGGIO, A.R. Relações entre parâmetros culturais e resposta espectral de cafezais. Pesquisa Agropecuária Brasileira, Brasília, v.29, n.3, p.379-386, 1994.

FAVARIN, J.L.; NETO, D.D.; GARCÍA, A.G.; NOVA, N.A. V.; FAVARIN, M.G.G.V. Equações para a estimativa do índice de área foliar do cafeeiro. Pesquisa Agropecuária Brasileira, Brasília, v.37, n.6, p.769-773, 2002.

IPPOLITI-RAMILO, G.A.; EPIPHANIO, J.C.N.; SHIMABUKURO, Y.E.; FORMAGGIO, A.R. Sensoriamento remoto orbital como meio auxiliar na previsão de safras. Agricultura em São Paulo, São Paulo, v.46, n.1, p.89-101, 1999.

LIU, W.T.H.; LIU, B.W.Y. Comparação entre três modelos de previsão de safra de café no Estado de Minas Gerais. Ciência e Cultura, Campinas, v.40, n.8, p.801-807, 1988.

MAZUCHELI, J.; ACHCAR, J.A. Algumas considerações em regressão não linear. Acta Scientiarum, Maringá, v.24, n.6, p.1.761-1.770, 2002.

MOREIRA, M.A.; ADAMI, M.; RUDORFF, B.F.T. Análise do comportamento espectral de café em imagens TM e ETM+ do Landsat 5 e 7. In: SIMPÓSIO BRASILEIRO DE SENSORIAMENTO REMOTO, 2003, Belo Horizonte. Anais... Belo Horizonte: INPE, 2003. p.189-196.

NASCIMENTO, C.R. Correção atmosférica de imagens do sensor AVHRR/NOAA utilizando produtos atmosféricos do sensor MODIS/TERRA. 2006. 129 f. Dissertação (Mestrado em 
Engenharia Agrícola) - Faculdade de Engenharia Agrícola, Universidade Estadual de Campinas, Campinas, 2006.

PÁSCUA, D.M.S. Cuantificación y valoración econômica Del servicio ambiental almacenamiento de carbono em sistemas agroflorestales de café em la Comarca Yassica Sur, Matagalpa,

Nicarágua. 2002. 131 f. Tese (Magister Scientiae) - Centro Agronómico Tropical de Investigación y Enseñanza, Turrialba, 2002.

PONZONI, F.J.; REZENDE, A.C.P. Caracterização espectral de estágios sucessionais de vegetação secundária arbórea em Altamira (PA), através de dados orbitais. Revista Árvore, Viçosa - MG, v.28, n.4, p.535-545, 2004.

RAMIREZ, G.M.; ZULLO JÚNIOR, J.; ASSAD, E.D.; PINTO, H.S. Comparação de dados dos satélites Ikonos-II e Landsat/ETM+ no estudo de áreas cafeeiras. Pesquisa Agropecuária Brasileira. Brasília, v.41, n.4, p.661-666, 2006.

WU, J.; WANG, D.; BAUER, M.E. Assessing broadband vegetation indices and QuickBird data in estimating leaf area index of corn and potato canopies. Field Crops Research, Amsterdam, v.102, n.1, 2007. Disponível em < http://www.sciencedirect.com>. Acesso em: 3 ago. 2008.

YI, J.L.R.; SHIMABUKURO, Y.E.; QUINTANILHA, J.A. Identificação e mapeamento de áreas de milho na região sul do Brasil utilizando imagens MODIS. Engenharia Agrícola, Jaboticabal, v.27, n.3, dez.2007. Disponível em <http://www.scielo.br/scielo.php?script=sci_arttext\&pid=S010069162007000400019\&lng=pt\&nrm=iso>. Acesso em: 28 dez. 2008.

ZULLO JÚNIOR, J. Correção atmosférica de imagens de satélite e aplicações. 1994. 190 f. Tese (Doutorado em Engenharia Elétrica) - Faculdade de Engenharia Elétrica, Universidade Estadual de Campinas, Campinas, 1994.

ZULLO JÚNIOR, J.; ARRUDA, F.B. Programa computacional para ajuste de equações em dados experimentais. Campinas: Instituto Agronômico, 1986. 23 p. (Boletim Técnico, 113) 